ISSN 0103-9954

\title{
ESTUDOS FITOSSOCIOLÓGICOS E DENDROMÉTRICOS EM UM FRAGMENTO DE CAATINGA, SÃO JOSÉ DE ESPINHARAS - PB
}

\author{
DENDROMETRIC AND PHYTOSOCIOLOGICAL STUDIES IN A FRAGMENT OF CAATINGA, \\ SÃO JOSÉ DE ESPINHARAS - PB
} Pierre Farias de Souza ${ }^{1}$ Josuel Arcanjo da Silva $^{2}$ Danielly da Silva Lucena ${ }^{3}$ William de Sousa Santos
Íkallo George Nunes Henriques ${ }^{4}$ Maria de Fátima de Araújo Lucena ${ }^{5} \quad$ Ademilson Daniel de Souza $^{4}$

\section{RESUMO}

O presente estudo teve como objetivos analisar a composição florística, caracterizar os parâmetros da estrutura interna da comunidade, ajustar modelos volumétricos para estimativas de volume de fustes, bem como estimar um fator de forma para fustes em um trecho de vegetação de Caatinga. A área de estudo se localiza no Assentamento Cachoeira, município de São José de Espinharas - PB. Foram utilizadas 49 parcelas de $400 \mathrm{~m}^{2}(20 \times 20 \mathrm{~m})$, sistematicamente distribuídas, com espaçamento entre parcelas de $300 \mathrm{x}$ $300 \mathrm{~m}$. Para a coleta dos dados de inventário, da regeneração e da estrutura interna (classe de vitalidade, qualidade de fuste e posição de copa), foi seguido o Protocolo de Medição da Rede de Manejo Florestal da Caatinga. Para o estudo das estimativas volumétricas, foi utilizada uma amostra 225 fustes, com medições da circunferência do caule a $0,30 \mathrm{~m}$ e $1,3 \mathrm{~m}$ do solo e altura total do maior fuste. Na cubagem rigorosa, o fuste foi medido em seções de $(1 \mathrm{~m})$, pelo método de Smalian adaptado. Os volumes totais por fuste foram obtidos com seis modelos lineares e não lineares, com duas opções para o diâmetro a $0,3 \mathrm{~m}$ e diâmetro equivalente. $\mathrm{O}$ fator de forma foi estimado pela razão entre o volume real por meio da cubagem rigorosa por fuste e o volume cilíndrico, cuja base foi a área seccional obtida pelo DAP a 1,30 m e altura do fuste. Foram encontradas, na área de estudo, 22 espécies, 11 famílias e 20 gêneros, sendo que a espécie Luetzelburgia bahiensis, teve seu primeiro registro na vegetação da Paraíba. A estrutura interna da comunidade indicou que acima de $71 \%$ dos fustes apresentaram-se saudáveis e $89,40 \%$ dos fustes apresentam baixa qualidade, ou seja, são fustes com ramificação, bifurcação e fustes muito tortos. Oito espécies apresentaram caule retilíneo e sem bifurcação e apenas $14,99 \%$ dos fustes fazem parte da classe dominante da floresta. Foi obtido o valor médio de 0,87 para o fator de forma estudado, porém, sugere-se o uso de dois fatores de forma para o cálculo de volume individual de fustes, fustes com diâmetro $<5 \mathrm{~cm}$ e com diâmetro $\geq 5 \mathrm{~cm}$, usar fatores de forma médios de 0,80 e 0,885 , respectivamente. Quatro modelos volumétricos apresentaram bons desempenhos para estimativas de volume de fustes, podendo assim ser usados para áreas com as mesmas semelhanças silviculturais na região.

Palavras-chave: composição florística; estrutura interna; modelos volumétricos.

\section{ABSTRACT}

The present study aimed to analyze the floristic composition, characterize the parameters of the internal

1 Engenheiro Florestal, Doutorando no Programa de Pós-Graduação em Ciências Florestais, Universidade de Brasília, Via L3 Norte, CEP 70910-900, Brasília (DF), Brasil. pierreflorestal@yahoo.com.br

2 Engenheiro Florestal, Dr., Unidade Acadêmica de Engenharia Florestal, Universidade Federal de Campina Grande, Av. Universitária, s/n, CEP 58708-110, Patos (PB), Brasil. jotaarcanjo@bol.com.br

3 Bióloga, Mestranda no Programa de Pós-Graduação em Biologia Vegetal, Universidade Federal de Pernambuco, Av. Professor Morais Rego, 1235, CEP 50670-901, Recife (PE), Brasil. botanicadane@gmail.com

4 Engenheiro Florestal, Unidade Acadêmica de Engenharia Florestal, Universidade Federal de Campina Grande, Av. Universitária, s/n, CEP 58708-110, Patos (PB), Brasil. william.ufcg@gmail.com/ ikallogeorge@yahoo.com.br/dsouzaig@gmail.com

5 Bióloga, Dra.., Unidade Acadêmica de Ciências Biológicas, Universidade Federal de Campina Grande, Av. Universitária, s/n, CEP 58708-110, Patos (PB), Brasil. fatimaarar@gmail.com

Recebido para publicação em 13/06/2012 e aceito em 9/03/2015 
structure, adjust volumetric models in order to obtain stem volume estimates as well as estimate a form factor for stems in a stretch of caatinga vegetation. The study area is located in 'Cachoeira' settlement, in the municipality of São José de Espinharas, PB state. Forty-nine samples, with an area of $400 \mathrm{~m}^{2}(20 \times 20 \mathrm{~m})$, were used and systematically distributed with the spacing between plots of $300 \times 300 \mathrm{~m}$. In order to collect the inventory data, regeneration and the internal structure (class of vitality, quality of stem and crown position), it was followed the Measurement Protocol of Caatinga Forest Management Network. For the study of volumetric estimates, it was used a 225 -sample stem, with measurements of the circumference of the stem at $0.30 \mathrm{~m}$ and $1.3 \mathrm{~m}$ of soil and overall height of the largest stem. In the rigorous scaling procedure, the stem was measured in $1 \mathrm{~m}$ sections by the adapted Smalian method. The total volumes by stem were obtained with six linear and nonlinear models, with two options for the diameter at $0.3 \mathrm{~m}$ and equivalent diameter. The form factor was estimated by the ratio between the actual volume obtained by stem rigorous scaling and cylindrical volume, which base was the sectional area obtained by the DAP at $1.30 \mathrm{~m}$ and height of the stem. We found, in the study area, 22 species, 11 families and 20 genera, and the species Luetzelburgia bahiensis, had its first record in the state of Paraiba. The internal structure of community indicated that above $71 \%$ of stems showed to be healthy and $89.40 \%$ of the stems have low quality, namely, they are stems with branch, fork and very askew stems. Eight species showed rectilinear stem and without fork and only $14.99 \%$ of the stems are part of the ruling class of the forest. The obtained value was 0.87 for the studied form factor, however, it suggests the use of two form factors for the calculation of individual volume stems, stems with a diameter $<5 \mathrm{~cm}$ and with a diameter $\geq 5 \mathrm{~cm}$, use medium form factor 0.80 and 0.885 , respectively. Four volumetric models showed good performance for volume estimates of stems. Therefore, they can be used for areas with the same silvicultural similarities in the same region.

Keywords: floristic composition; internal structure; volumetric models.

\section{INTRODUÇÃO}

O Bioma Caatinga possui uma área aproximada de $826.411 \mathrm{~km}^{2}$ e se estende pelos Estados do Ceará, Bahia, Paraíba, Pernambuco, Piauí e do Rio Grande do Norte, Alagoas, além de pequenas porções de Minas Gerais e do Maranhão (IBAMA, 2010), correspondendo, aproximadamente, a $54 \%$ da região Nordeste e $11 \%$ do território brasileiro. Segundo Rodal et al. (2008), a Caatinga apresenta uma variada cobertura vegetal, cuja distribuição é determinada pelo clima, relevo e embasamento geológico que, em suas múltiplas inter-relações, resultam em ambientes ecológicos bastante variados.

A análise da composição florística de remanescentes de Caatinga é importante para a caracterização das diferentes fácies, constituindo-se como ferramenta para o entendimento de aspectos da ecologia regional (MARANGON et al., 2007), ressaltado que, de forma geral, mesmo as áreas mais bem conservadas da região apresentam indícios de exploração pretérita. A estrutura arbórea propicia informações a respeito do grau de perturbação e do estágio de desenvolvimento sucessional em que essa comunidade se encontra (ALVES JUNIOR et al., 2007).
Existe a necessidade da análise de quantificação e qualificação do estoque madeireiro das florestas nativas, isso leva a buscar métodos eficientes de estimativa do volume comercial e, em relação à qualidade, o conhecimento da estrutura interna das árvores, ou seja, a vitalidade, qualidade do fuste e a posição no dossel. Essas informações norteiam decisões silviculturais que culminam no aumento da produção e rentabilidade da floresta (THAINES et al., 2010).

Equações de volume vêm sendo desenvolvidos para quantificar o volume de árvores individuais. Dentre as metodologias que possibilitam obtenção destes volumes, pode ser citada: a observação das equações de volume por árvores individuais para reduzir custos dos levantamentos florestais sem a perda da eficiência, podendo utilizar equações de volume por unidade de área (SCOLFORO et al., 1994).

As estimativas volumétricas de espécies arbóreas compõem um dos objetivos do inventário florestal. Os volumes podem ser expressos em função do diâmetro, da altura e do fator de forma. As estimativas possuem precisão devido ao uso de modelos volumétricos, por local, região e formação vegetal (SILVA et al., 2000).

Poucas referências são encontradas 
relativas à modelagem volumétrica de madeira das fitofisionomias em áreas de Caatinga (FERRAZ, 2011; ALVES JUNIOR, 2010; SAMPAIO et al., 2010; SILVA, 2005; ARAÚJO et al., 2004).

Isso tem induzido à pesquisa e ao desenvolvimento de modelos estatísticos aplicados ao manejo de florestas naturais que auxiliem na quantificação de precisão dessas madeiras e de transformação em produto final, com o intuito de torná-las mais rentáveis (CHICHORRO et al., 2003)

O presente trabalho objetiva analisar a composição florística, caracterizar os parâmetros da estrutura interna e ajustar modelos volumétricos para estimativas de volume de fustes, bem como estimar um fator de forma para fustes na vegetação de Caatinga para a área de estudo.

\section{MATERIAIS E MÉTODOS}

Área de Estudo

O estudo foi desenvolvido na reserva legal do "Assentamento Cachoeira", localizado no município de São José de Espinharas, Paraíba, inserido nas seguintes coordenadas geográficas: 6 $48^{\prime} 33,11^{\prime \prime}, 6^{\circ} 47^{\prime} 09,94^{\prime \prime}$ de latitude sul, e $37^{\circ} 19^{\prime} 53,64$ ", $37^{\circ} 19^{\prime} 23,66^{\prime \prime}$ de longitude oeste e apresenta uma área de aproximadamente 430 hectares, distante aproximadamente $6 \mathrm{~km}$ da zona urbana do referido município.

O município de São José de Espinharas situa-se no Polígono das Secas. Segundo a divisão do Estado da Paraíba em regiões bioclimáticas, o município enquadra-se no bioclima $4 \mathrm{aTh}$ - tropical quente, com seca acentuada. Segundo Köppen, a predominância é do tipo Bsh, com presença de semiaridez. A temperatura média anual situa-se em torno de $28^{\circ} \mathrm{C}$. A precipitação média para o período de 1911-1990 no município foi de 848,6 mm/ano (UNIVERSIDADE FEDERAL DE CAMPINA GRANDE, 2014), sendo irregularmente distribuídas, com $79 \%$ do total concentrada em 4 meses do ano (BELTRÃO et al., 2005).

Os solos predominantes são classificados como Luvissolos (EMBRAPA SOLOS, 2006). A topografia apresenta, na maior parte da área do município, relevo ondulado a suavemente ondulado, com exceção de áreas situadas na porção sudeste, de relevo ondulado a fortemente ondulado (BELTRÃO et al., 2005). Segundo a classificação de Veloso et al. (1991), a vegetação é caatinga arbustiva arbórea.

\section{Amostragem e coleta de dados}

\section{Inventário florestal}

No inventário da área, foram utilizadas 49 parcelas, com área de $400 \mathrm{~m}^{2}(20 \times 20 \mathrm{~m})$, representando uma área amostral de 1,96 ha, as parcelas foram sistematicamente distribuídas, com espaçamento entre parcelas de 300 x 300 $\mathrm{m}$, demarcadas com auxílio de GPS (Global Position System) de navegação. Nas parcelas, foram amostrados todos os indivíduos lenhosos vivos ou mortos ainda em pé, cuja circunferência do caule a $1,30 \mathrm{~m}$ de altura fosse superior a $6 \mathrm{~cm}$, seguindo o Protocolo de Medições de Parcelas Permanentes (REDE DE MANEJO FLORESTAL DA CAATINGA, 2005).

O material botânico fértil ou estéril foi coletado e identificado por especialistas e por comparação com material de herbário, o sistema de classificação utilizado foi o APG III (REVEAL, CHASE, 2011). As exsicatas foram depositadas no Herbário do Centro de Saúde e Tecnologia Rural, Universidade Federal de Campina Grande.

\section{Autorização ambiental}

Para que o estudo de modelagem fosse desenvolvido na área de reserva legal, foi encaminhado à Superintendência de Administração do Meio Ambiente (SUDEMA) um pedido de Autorização Ambiental para a coleta de dados do inventário florestal e cubagem rigorosa, concedida por meio do processo $\mathrm{n}^{\circ}$ 2011-002601/TEC/AA0047.

\section{Estrutura interna}

Para o estudo de estrutura interna da comunidade arbórea, foram considerados os seguintes atributos: classe de vitalidade, qualidade do fuste e posição da copa, seguindo o Protocolo de Medições de Parcelas Permanentes (REDE DE MANEJO FLORESTAL DA CAATINGA, 2005).

Para o estudo da classe de vitalidade, cada fuste foi classificado em: 1 = fuste sadio; 2 = fuste doente ou muito atacado por insetos ou patógenos; 3 = fuste morto. Para o estudo da qualidade do fuste, registrou-se a codificação ou o estado que prevalece a maior parte do fuste, usando a seguinte codificação: 1 = totalmente reto, sem defeitos, sem bifurcações até $2,50 \mathrm{~m} ; 2=$ ligeiramente torto ou 
com poucos defeitos, sem bifurcações até $2,50 \mathrm{~m}$; 3 = muito torto, com defeitos graves (oco, rachado, podre), ou com bifurcações até $2,50 \mathrm{~m}$. A posição da copa de cada indivíduo foi avaliada em função do dossel geral na parcela e não apenas das árvores vizinhas, pelo uso da seguinte codificação: $1=$ dominante (se pertence aos $10 \%$ dos fustes mais altos da parcela); 2 = intermediária (localizada no nível médio de altura dos fustes da parcela); $3=$ oprimida (fustes que se localizada por debaixo de outras copas da parcela).

\section{Estimativas Dendrométricas}

\section{Cubagens de fustes}

Para o estudo da cubagem rigorosa, foi utilizada uma amostra com 225 fustes, de 10 espécies florestais existentes no Bioma Caatinga: Anadenanthera colubrina, Aspidosperma pyrifolium, Bauhinia cheilantha, Combretum sp., Croton blanchetianus, Erythroxylum caatinga, Handroanthus impetiginosus, Mimosa ophthalmocentra, Mimosa tenuiflora e Poincianella pyramidalis. Estas espécies foram distribuídas em oito classes diamétricas, em que se procurou amostrar um número de árvores aproximadamente igual por classe de diâmetro.

Para cada fuste cubado, foram tomadas as medidas de circunferência do caule a $0,30 \mathrm{~m}$ $\left(\mathrm{CAP}_{0,30}\right)$ e $1,3 \mathrm{~m}\left(\mathrm{CAP}_{1,30}\right)$ do solo. Após o corte do fuste, tomou-se como altura total a maior bifurcação para a medição. As árvores foram agrupadas em classes diamétricas com amplitude de $5 \mathrm{~cm}$, considerando como referência a $\mathrm{C}_{0,30}$.

A árvore foi medida em seções de $(1 \mathrm{~m})$ ao longo dos fustes e galhos, tomando a circunferência nas duas extremidades até um valor mínimo aproveitável em torno de $1,5 \mathrm{~cm}$ de diâmetro, anotando-se, quando fosse o caso, a circunferência e o comprimento fracionário da seção final.

Após a medição da circunferência nas duas extremidades de cada seção (tora) e do seu respectivo comprimento, os volumes totais por árvore foram obtidos através da fórmula de Smalian (CAMPOS; LEITE, 2009), pelas seguintes expressões:

$$
V_{j}=\sum_{i=1}^{n}\left(\frac{g_{i 1}+g_{i 2}}{2}\right) \cdot L_{i} ; g_{i}=\left(\frac{\partial \cdot D_{i}^{2}}{40.000}\right)
$$

Em que: Vj é o volume do fuste $\mathrm{j}$, com casca, em $\mathrm{m}^{3}(\mathrm{j}=1,2, \ldots, \mathrm{m})$, gi é a área da seção $i$, em $\mathrm{m}^{2}$ ( $i_{1}$ é o início da seção e $i_{2}$ é o fim da seção, i parte de $1,2, \ldots, n)$, Di é o diâmetro da seção i, em $\mathrm{cm}$, Li é o comprimento da seção i, em $\mathrm{m}$ e $\pi$ é 3,141592653589.

\section{Ajuste de modelos volumétricos}

Para estimar os volumes com casca, incluindo os galhos com o diâmetro mínimo aproveitável, de cada

planta lenhosa, por classe diamétrica e por espécie, com dados provenientes da cubagem rigorosa, foram utilizados dois modelos na forma linearizada: Schumacher e Hall (1 e 3), o modelo de Spurr (2 e $4)$, e o modelo de Schumacher e Hall na forma não linear (5 e 6), todas conforme descrito por Campos e Leite (2009) (Tabela 1).

Para cada modelo testado, foram utilizadas duas opções para a variável independente diâmetro: diâmetro 0,3 metros de altura do solo, seguindo o Protocolo de Medições de Parcelas Permanentes (REDE DE MANEJO FLORESTAL DA CAATINGA, 2005), e diâmetro equivalente (DEq) a 1,3 metros de altura (SILVA, 2005).

Estas opções para a variável independente diferem de Campos e Leite (2009), que apresentam apenas a variável DAP a 1,3 metros. O uso do DEq é necessário para o estudo de algumas espécies da Caatinga que apresentam fuste ramificado, enquanto que para os fustes que não apresentam bifurcação, $o$ $\mathrm{DEq}$ foi o próprio DAP.

Como, na Caatinga, muitos dos fustes possuem mais de uma ramificação na altura de 1,30 (bifurcação, trifurcação e até mais galhos), o diâmetro equivalente foi obtido pela seguinte expressão:

$$
D E q=\sqrt{\sum_{D A P}^{2}}
$$

Em que DEq é o diâmetro equivalente, em $\mathrm{cm}$ e DAP é o diâmetro com casca medido a $1,30 \mathrm{~m}$ do solo, em $\mathrm{cm}$.

O diâmetro equivalente é uma variável calculada nos estudos florestais. Esta variável não existe na floresta, porém, para que a mesma possa ser mensurada, é necessário que uma planta possua, no mínimo, uma bifurcação a uma altura de 1,30 metros. 
TABELA 1: Modelos volumétricos de dupla entrada.

TABLE 1: Volumetric models of double entry.

\begin{tabular}{cc}
\hline 1 & Modelos \\
\hline 2 & $\operatorname{Ln}\left(\mathrm{Vt}_{\mathrm{j}}\right)=\beta_{0}+\beta_{1} \cdot \operatorname{Ln}\left(\mathrm{D}_{0,3 \mathrm{~m}(\mathrm{j})}\right)+\beta_{2} \cdot \operatorname{Ln}\left(\mathrm{Ht}_{\mathrm{j}}\right)+\varepsilon_{\mathrm{j}}$ \\
3 & $\operatorname{Ln}\left(\mathrm{Vt}_{\mathrm{j}}\right)=\beta_{0}+\beta_{1} \cdot \operatorname{Ln}\left(\mathrm{D}_{0,3 \mathrm{~m}(\mathrm{j})}^{2} \cdot \mathrm{Ht}_{\mathrm{j}}\right)+\varepsilon_{\mathrm{j}}$ \\
4 & $\operatorname{Ln}\left(\mathrm{Vt}_{\mathrm{j}}\right)=\beta_{0}+\beta_{1} \cdot \operatorname{Ln}\left(\mathrm{DEq}_{\mathrm{j}}\right)+\beta_{2} \cdot \operatorname{Ln}\left(\mathrm{Ht}_{\mathrm{j}}\right)+\varepsilon_{\mathrm{j}}$ \\
5 & $\operatorname{Ln}\left(\mathrm{Vt}_{\mathrm{j}}\right)=\beta_{0}+\beta_{1} \cdot \operatorname{Ln}\left(\mathrm{DEq}_{\mathrm{j}}^{2} \cdot \mathrm{Ht}_{\mathrm{j}}\right)+\varepsilon_{\mathrm{j}}$ \\
6 & $\mathrm{Vt}_{\mathrm{j}}=\beta_{0} \cdot\left(\mathrm{DEq}^{\beta_{1}}\right) \cdot\left(\mathrm{Ht}_{\mathrm{j}}{ }^{\beta_{2}}\right)$ \\
$\mathrm{Vt}=\beta_{0} \cdot\left(\mathrm{D}_{0,3 \mathrm{~m}(\mathrm{j})} \beta_{1}\right) \cdot\left(\mathrm{Ht}_{\mathrm{j}}{ }^{\beta_{2}}\right)$
\end{tabular}

Em que: $\mathrm{Vtj}=$ volume total com casca, em m ${ }^{3} ; \mathrm{D}_{0,3 \mathrm{~m}(\mathrm{i})}=$ diâmetro a altura da base a $0,3 \mathrm{~m}$, em cm; $\mathrm{DEq}_{\mathrm{i}}=$ diâmetro equivalente, em cm; $\mathrm{Htj}=$ altura total da maior ramificação, em $\mathrm{m} ; \beta_{0}, \beta_{1}$ e $\beta_{2}=$ Coeficientes dos modelos a serem estimados; $\varepsilon j$ = erro aleatório; $\mathrm{Ln}=$ logaritmo neperiano.

\section{Critérios para a avaliação da qualidade dos ajustes}

Como critérios para a avaliação da qualidade dos ajustes dos modelos gerados e para a escolha da melhor equação, foram utilizados o coeficiente de determinação ajustado, erro padrão residual da estimativa e a análise gráfica dos resíduos em porcentagem (SILVA, 2005).

Foram calculados o coeficiente de determinação ajustado e o erro padrão da estimativa, seguindo as expressões utilizadas por Miguel et al. (2010), para que se pudessem fazer comparações entre os modelos lineares e não lineares, através das expressões:

$$
\mathrm{R}_{\dot{\mathbf{j}} .}^{2}=1-\left(\frac{\mathrm{n}-1}{\mathrm{n}-\mathrm{p}-1}\right) \cdot \frac{\text { SQres }}{\text { SQtot }}
$$

Em que $\mathrm{R}_{\text {aj }}^{2}$ é o coeficiente de determinação ajustado, n é o número de observações (tamanho da amostra), p é o número de variáveis independentes do modelo matemático em questão, SQres é a Soma de quadrado do resíduo e SQtot é a Soma de quadrado total.

O índice de Schlaegel (IA) e uma estatística comparável ao $\mathrm{R}^{2}{ }_{\text {aj. }}$, quando se trata de equações aritméticas, portanto, este índice serve como um comparador de equações de diferentes naturezas
(MIGUEL et al., 2010)

O erro padrão da estimativa - (Syx) e em percentagem (Syx\%), quanto mais próximo de zero, mais preciso, pois o valor indica o quão próximos os valores estimados estão dos valores observados.

$$
S_{\mathrm{y}}=\sqrt{\frac{\sum\left(\mathrm{X}_{\mathrm{i}}-\mathrm{Y}_{\mathrm{i}}\right)^{2}}{\mathrm{n}-\mathrm{p}-1}} \mathrm{Syx} \%=\frac{\mathrm{Syx}}{\mathrm{h}} \cdot 100
$$

Em que $S_{x y}$ é o erro padrão da estimativa, Syx\% é o erro padrã̃o da estimativa em porcentagem, $\mathrm{X}_{\mathrm{i}}$ é o volume observado em i-ésimo fuste, $\mathrm{Y}_{\mathrm{i}}$ é o volume estimado em i-ésimo fuste, Ym é o volume médio observado, $\mathrm{n}$ é o número de observações (tamanho da amostra) e p é o número de coeficientes do modelo matemático.

Os resíduos, depois de calculados, foram distribuídos em um gráfico cartesiano, onde nas abscissas, foi projetado a distribuição dos fustes em classes diamétricas e, no eixo das ordenadas, os resíduos em porcentagem, calculados pela seguinte expressão:

$$
\text { R } \mathrm{s} \%=\frac{(\text { VEst }- \text { VObs })}{\text { VObs }} \cdot 100
$$

Em que: Res\% é o resíduo em porcentagem, VEst é o volume estimado pela equação matemática, $\mathrm{em} \mathrm{m}^{3} \mathrm{e}$ VObs é o volume obtido através da cubagem 
rigorosa, em $\mathrm{m}^{3}$.

\section{Fator de forma}

Esta estimativa foi obtida pela razão entre o volume real obtido da cubagem rigorosa por fuste e o volume cilíndrico, cuja base é a área obtida pelo DAP a $1,30 \mathrm{~m}$ e altura total do fuste (SOARES et al., 2006), dado através da seguinte expressão.

$$
\mathrm{f}=\frac{\text { VObs }}{\text { VEst }}
$$

Em que $f$ é o fator de forma, VObs é o volume obtido da cubagem rigorosa e VEst é o volume obtido pelo DAP ou DEq e pela altura da árvore.

Segundo Silva (2005), o fator de forma para espécies da Caatinga tem o significado de um fator de conversão, em que o volume total da árvore é todo volume aproveitável, incluindo o volume do fuste e dos galhos com diâmetro até próximo de 1,5 $\mathrm{cm}$.

\section{Análises dos dados}

$\mathrm{O}$ processamento $\mathrm{e}$ as análises foram realizados utilizando-se os softwares Statistica 5 e Mata Nativa 2 (FUNDAÇÃO DE CIÊNCIA E TECNOLOGIA, 2006).

\section{RESULTADOS E DISCUSSÃO}

\section{Levantamento florístico}

No levantamento da vegetação arbustiva arbórea, foram identificadas 22 espécies pertencentes a 11 famílias botânicas e 20 gêneros (Tabela 2).

A grande família das Fabaceas contribuiu com um total de dez espécies lenhosas. As famílias Euphorbiaceae e Bignoniaceae contribuíram cada uma com duas espécies florestais arbustivas arbóreas. As demais famílias: Anacardiaceae, Apocynaceae, Burseraceae, Combretaceae, Erythroxylaceae, Malvaceae, Rhamnaceae e Verbenaceae contribuíram apenas com uma espécie (Tabela 2).

As famílias Fabaceae, Euphorbiaceae e Bignoniaceae representam mais de $63 \%$ da riqueza das espécies arbustivo-arbóreas presentes na área de estudo. Esses resultados corroboram com Oliveira et al (2009) que analisaram quatro áreas de Caatinga do Cariri paraibano e encontraram como as famílias mais representantes Fabaceae e Euphorbiaceae.

Estas famílias também foram registradas em diferentes áreas de Caatinga (SANTANA; SOUTO, 2006; GUEDES et al., 2012; SILVA, 2005; ARAÚJO, 2007; ANDRADE et al., 2009; ALMEIDA NETO et al., 2009). Esses resultados demonstram que as espécies destas famílias estão distribuídas ao longo da Caatinga.

A espécie Luetzelburgia bahiensis teve seu primeiro registro de ocorrência para o estado da Paraíba, de acordo com informações disponíveis na base de dados Lista de Espécies da Flora do Brasil (BFG, 2015), esta espécie ocorre somente em áreas de Caatinga nos estados da Bahia, Pernambuco e Piauí.

\section{Estrutura interna}

A análise da vitalidade dos fustes de espécies lenhosas da Caatinga demonstrou que $71,73 \%$ dos fustes apresentaram-se como saudáveis (Tabela 3). Na vegetação de caatinga localizada em área de serra, Leite (2010) registrou $91,2 \%$ do total de indivíduos amostrados como sadios.

As espécies Anadenanthera colubrina, Aspidosperma. pyrifolium, Croton blanchetianus e Mimosa ophthalmocentra apresentaram maior número de fustes sadios $92,67 \%, 81,74 \%, 91,20 \%$ e $80,51 \%$, respectivamente (Tabela 3). Por outro lado, as espécies Poincianella pyramidalis, Mimosa tenuiflora, Combretum sp. e Bauhinia cheilantha apresentaram maior número de fustes doentes ou muito atacados, por insetos e patógenos $44,56 \%$; $40,72 \% ; \quad 28,36 \%$ e $39,86 \%$, respectivamente (Tabela 3).

$\mathrm{Na}$ análise de qualidade de fuste, apenas as espécies: Anadenanthera colubrina, Poincianella pyramidalis, Aspidosperma pyrifolium, Mimosa tenuiflora, Combretum sp., Handroanthus impetiginosus, Luetzelburgia bahiensis, Croton blanchetianus e Mimosa ophthalmocentra apresentaram fustes retilíneos e sem bifurcação até $2,5 \mathrm{~m}$ de altura (Tabela 4). Por outro lado, as espécies Croton blanchetianus, Poincianella pyramidalis, Combretum sp., Mimosa tenuiflora e Aspidosperma pyrifolium apresentaram as maiores porcentagens de fustes com bifurcação abaixo de $2,5 \mathrm{~m}$ de altura, $87,78 \%, 96,33 \%, 83,25 \%, 88,15 \%$, $94,51 \%$, respectivamente (Tabela 4).

A densidade de fustes da classe I não foi superior à densidade de fustes da classe II, e o somatório das mesmas não foi superior à densidade 
TABELA 2: Florística das espécies arbóreas da Reserva Legal do Assentamento Cachoeira, São José de Espinharas - PB, listadas por ordem alfabética de famílias.

TABLE 2: $\quad$ Floristic of tree species in Legal Reserve Settlement 'Cachoeira', São José de Espinharas PB state, listed alphabetically by families.

\begin{tabular}{|c|c|}
\hline Família/Espécie & Nome Comum \\
\hline \multicolumn{2}{|l|}{ Anacardiaceae } \\
\hline Myracrodrum urundeuva Allem. & Aroeira \\
\hline \multicolumn{2}{|l|}{ Apocynaceae } \\
\hline Aspidosperma pyrifolium Mart. & Pereiro \\
\hline \multicolumn{2}{|l|}{ Bignoniaceae } \\
\hline Handroanthus impetiginosus (Mart. ex. Dc) Mattos & Ipê-roxo \\
\hline Fridericia dichotoma (Jacq.) L.G.Lohmann & Cipó-cruz \\
\hline \multicolumn{2}{|l|}{ Burseraceae } \\
\hline Commiphora leptophloeos (Mart.) J. B. Gillett & Imburana-de-cambão \\
\hline \multicolumn{2}{|l|}{ Combretaceae } \\
\hline Combretum sp. & Mofumbo \\
\hline \multicolumn{2}{|l|}{ Erythroxylaceae } \\
\hline Erythroxylum caatinga Plowman & Rompe-gibão \\
\hline \multicolumn{2}{|l|}{ Euphorbiaceae } \\
\hline Croton blanchetianus Baill. & Marmeleiro \\
\hline Jatropha mollissima (Pohl) Baill. & Peão-bravo \\
\hline \multicolumn{2}{|l|}{ Fabaceae } \\
\hline Amburana cearensis (Allemão) A. C. Sm. & Cumaru \\
\hline Anadenanthera colubrina (Vell.) Brenan. & Angico \\
\hline Bauhinia cheilantha (Bong.) Steud. & Mororó \\
\hline Libidibia ferrea (Mart. exTul.) L. P. Queiroz, comb. nov. & Jucá \\
\hline Luetzelburgia bahiensis Yakovlev. & Pau-pedra \\
\hline Mimosa acutistipula (Mart.) Benth. & Jurema-vermelha \\
\hline Mimosa ophthalmocentra Mart. exBenth. & Jurema-branca \\
\hline Mimosa tenuiflora (Willd.) Poir. & Jurema-preta \\
\hline Poincianella pyramidalis (Tul.) L. P. Queiroz, comb. nov. & Catingueira \\
\hline Senna spectabilis (DC.) H.S.Irwin \& Barneby & Canafístola \\
\hline \multicolumn{2}{|l|}{ Malvaceae } \\
\hline Pseudobombax marginatum (A.St. - Hil., Juss. \&Cambess.) A. Robyns & Embiratanha \\
\hline \multicolumn{2}{|l|}{ Rhamnaceae } \\
\hline Ziziphus Joazeiro Mart. & Juazeiro \\
\hline \multicolumn{2}{|l|}{ Verbenaceae } \\
\hline Lantana camara $\mathrm{L}$. & Chumbinho \\
\hline
\end{tabular}

de fustes da classe III. Esta última classe foi representada por um total de $89,40 \%$ de fustes muito tortos, com defeitos graves (oco, rachado, podre), ou com bifurcações até $2,5 \mathrm{~m}$ de altura (Tabela 4). Estes resultados mostram um baixo potencial de utilização das espécies lenhosas da área de estudo para fins mais nobres como móveis e madeira para serraria.

Como $71,73 \%$ da densidade dos fustes apresentam-se saudáveis e $89,40 \%$ dos fustes apresentam uma qualidade de fuste classe III, deduzse que a grande maioria dos fustes são saudáveis e apresentam ramificação, bifurcação abaixo de $2,5 \mathrm{~m}$ e que são fustes muito tortos, típico de vegetação de 
TABELA 3: Densidade absoluta $\left(h^{-1}\right)$ e relativa das espécies nas classes de vitalidade e de posição do dossel em um trecho de caatinga, São José de Espinharas - PB.

TABLE 3: Absolute density $\left(\mathrm{ha}^{-1}\right)$ and relative (\%) of species in vitality and canopy position classes in a stretch of caatinga, São José de Espinharas - PB state.

\begin{tabular}{|c|c|c|c|c|c|c|c|c|}
\hline \multirow{2}{*}{ Nome Científico } & \multicolumn{4}{|c|}{ Vitalidade } & \multicolumn{4}{|c|}{ Posição do Dossel } \\
\hline & C I & C II & C III & Total & C I & C II & C III & Total \\
\hline Anadenanthera colubrina & 70,92 & 5,61 & - & 76,53 & 53,57 & 8,67 & 14,29 & 76,53 \\
\hline Myracrodrum urundeuva & 6,63 & 0,51 & - & 7,14 & 4,08 & 0,51 & 2,55 & 7,14 \\
\hline Poincianella pyramidalis & 336,74 & 273,47 & 3,57 & 613,78 & 78,07 & 131,63 & 404,08 & 613,78 \\
\hline Aspidosperma pyrifolium & 205,61 & 45,92 & - & 251,53 & 32,14 & 35,71 & 183,67 & 251,53 \\
\hline Mimosa tenuiflora & 185,71 & 133,16 & 8,16 & 327,04 & 82,65 & 100,00 & 144,39 & 327,04 \\
\hline Erythroxylum caatinga & 42,35 & 11,74 & 0,51 & 54,59 & 1,02 & 4,08 & 49,49 & 54,59 \\
\hline Combretum sp. & 267,35 & 108,67 & 7,14 & 383,16 & 61,74 & 62,25 & 259,18 & 383,16 \\
\hline Bauhinia cheilantha & 43,37 & 29,08 & 0,51 & 72,96 & 2,55 & 10,71 & 59,69 & 72,96 \\
\hline Pseudobombax marginatum & 1,02 & - & - & 1,02 & - & - & 1,02 & 1,02 \\
\hline Commiphora leptophloeos & 9,69 & - & - & 9,69 & 0,51 & 1,53 & 7,65 & 9,69 \\
\hline Handroanthus impetiginosus & 38,27 & - & - & 38,27 & 14,80 & 6,63 & 16,84 & 38,27 \\
\hline Luetzelburgia bahiensis & 0,51 & - & - & 0,51 & 0,51 & - & - & 0,51 \\
\hline Croton blanchetianus & 708,67 & 63,78 & 4,59 & 777,04 & 46,94 & 130,61 & 599,49 & 777,04 \\
\hline Cynophalla flexuosa & 1,53 & 1,53 & - & 3,06 & 0,51 & - & 2,55 & 3,06 \\
\hline Lantana camara & 6,63 & 1,53 & - & 8,16 & 0,51 & - & 7,65 & 8,16 \\
\hline Libidibia ferrea & 2,55 & - & - & 2,55 & 1,02 & - & 1,53 & 2,55 \\
\hline Ziziphus joazeiro & 3,57 & - & - & 3,57 & 2,55 & 0,51 & 0,51 & 3,57 \\
\hline Morta & - & - & 90,31 & 90,31 & - & 1,53 & 88,78 & 90,31 \\
\hline Mimosa ophthalmocentra & 162,25 & 39,29 & - & 201,53 & 56,12 & 88,27 & 57,14 & 201,53 \\
\hline Mimosa acutistipula & - & 1,02 & - & 1,02 & 1,02 & - & - & 1,02 \\
\hline Amburana cearenses & 1,02 & - & - & 1,02 & 0,51 & - & 0,51 & 1,02 \\
\hline Jatropha molissima & 13,78 & 1,02 & - & 14,80 & - & 0,51 & 14,29 & 14,80 \\
\hline Senna spectabilis & 0,51 & - & - & 0,51 & - & - & 0,51 & 0,51 \\
\hline Total Absoluto & 2108,67 & 716,33 & 114,80 & 2939,80 & 440,82 & 583,16 & 1915,82 & 2939,80 \\
\hline Total Relativo & 71,73 & 24,36 & 3,91 & 100,00 & 14,99 & 19,84 & 65,17 & 100,00 \\
\hline
\end{tabular}

Em que: vitalidade - Classe I = Fuste Sadio; Classe II = Fuste doente ou muito atacado por insetos ou patógenos; Classe III = Fuste morto; posição do dossel - Classe I $=$ Dominante, $10 \%$ dos fustes + altas da parcela; Classe II $=$ Intermediária, nível médio dos fustes da parcela; Classe III = Oprimida $=$ Fustes localizados debaixo de outras copas da parcela.

caatinga.

Apenas as espécies Anadenanthera colubrina, Poincianella pyramidalis, Mimosa tenuiflora, Combretum sp., Croton blanchetianus e Mimosa ophthalmocentra apresentaram o maior número de fustes presentes na classe dominante do dossel da floresta. Estas espécies fazem parte do estrato superior da estrutura vertical na área de Caatinga estudada (Tabela 4).

Leite (2010) registrou em uma área de caatinga fustes retos e sem bifurcação representando
$50 \%$ do total, fustes tortos e com bifurcações representaram 35,2\% dos indivíduos e $14,8 \%$ dos indivíduos apresentaram fustes ligeiramente tortos ou pouco defeituosos e sem bifurcações. Segundo o autor, esses dados foram determinados pela quantidade de indivíduos de Croton sonderianus, que geralmente apresenta fustes retos e sem bifurcação.

Em relação à posição do dossel, a classe oprimida apresentou o maior número de indivíduos, com uma porcentagem de $65,17 \%$ dos fustes, a 
TABELA 4: Densidade absoluta $\left(\right.$ ha $\left.^{-1}\right)$ e relativa das espécies na classe de qualidade de fustes em trecho de caatinga, São José de Espinharas - PB.

TABLE 4: Absolute density $\left(\mathrm{ha}^{-1}\right)$ and relative (\%) of species in stems quality class in a caatinga stretch, São José de Espinharas - PB state.

\begin{tabular}{|c|c|c|c|c|}
\hline \multirow{2}{*}{ Nome Científico } & \multicolumn{4}{|c|}{ Qualidade de Fuste } \\
\hline & $\mathrm{C} \mathrm{I}$ & C II & C III & Total \\
\hline Anadenanthera colubrina & 12,76 & 25,00 & 38,78 & 76,53 \\
\hline Myracrodrum urundeuva & - & - & 7,14 & 7,14 \\
\hline Poincianella pyramidalis & 3,57 & 18,88 & 589,29 & 611,74 \\
\hline Aspidosperma pyrifolium & 3,57 & 10,20 & 237,25 & 251,02 \\
\hline Mimosa tenuiflora & 4,59 & 33,67 & 284,69 & 322,96 \\
\hline Erythroxylum caatinga & - & 1,02 & 52,55 & 53,57 \\
\hline Combretum sp. & 4,59 & 59,18 & 316,84 & 380,61 \\
\hline Bauhinia cheilantha & - & 5,61 & 67,35 & 72,96 \\
\hline Pseudobombax marginatum & - & - & 1,02 & 1,02 \\
\hline Commiphora leptophloeos & - & - & 9,69 & 9,69 \\
\hline Handroanthus impetiginosus & 0,51 & 6,63 & 31,12 & 38,27 \\
\hline Luetzelburgia bahiensis & 0,51 & - & - & 0,51 \\
\hline Croton blanchetianus & 20,41 & 73,98 & 678,06 & 772,45 \\
\hline Cynophalla flexuosa & - & 0,51 & 2,55 & 3,06 \\
\hline Lantana camara & - & - & 8,16 & 8,16 \\
\hline Libidibia ferrea & - & - & 2,55 & 2,55 \\
\hline Ziziphus joazeiro & - & 0,50 & 3,06 & 3,57 \\
\hline Mimosa ophthalmocentra & 3,06 & 11,70 & 186,74 & 201,53 \\
\hline Mimosa acutistipula & - & - & 1,02 & 1,02 \\
\hline Amburana cearenses & - & - & 1,02 & 1,02 \\
\hline Jatropha molissima & - & - & 14,80 & 14,80 \\
\hline Senna spectabilis & - & - & 0,51 & 0,51 \\
\hline Total Absoluto & 53,57 & 246,94 & 2534,18 & 2834,69 \\
\hline Total Relativo & 1,89 & 8,71 & 89,40 & 100,00 \\
\hline
\end{tabular}

Em que: qualidade do fuste - Classe I $=$ Totalmente reto, sem bifurcação até $2,5 \mathrm{~m}$; Classe II $=$ Ligeiramente torto ou com poucos defeitos, sem bifurcações até 2,5 m; Classe III = Muito torto, com defeitos graves (oco, rachado, podre), ou com bifurcações até $2,5 \mathrm{~m}$.

classe intermediária apresentou $19,84 \%$ dos fustes e a classe dominante, $14,99 \%$ dos fustes (Tabela 3 ).

\section{Modelos Volumétricos - Estimativas dos parâmetros}

As estimativas entre os $\mathrm{R}^{2}$ ajustados para os modelos utilizados apresentaram melhores ajustes para os modelos lineares I a IV (Tabela 5). Para as estimativas das diferenças entre o erro padrão da estimativa (Syx) para os modelos, houve uma variação entre $4,51 \%$ a $24,66 \%$, sendo que os modelos II e III, apresentaram as maiores diferenças quando comparados com o modelo VI. Por esses critérios, a escolha da melhor equação expressa a melhor distribuição gráfica dos resíduos (Tabela 5).

Em relação à distribuição dos resíduos percentuais para as estimativas de volume total as equações dos modelos I e II com a opção para a 
TABELA 5: Modelos volumétricos com as equações ajustadas para uma vegetação de caatinga em São José de Espinharas - PB.

TABLE 5: Volumetric models with equations adjusted for a caatinga vegetation in São Jose de Espinharas - PB state.

\begin{tabular}{|c|c|c|c|}
\hline & Equações Ajustadas & $\begin{array}{l}\text { Syx } \\
(\%)\end{array}$ & $\mathrm{R}^{2} \mathrm{Aj}$. \\
\hline 1 & $\operatorname{Ln}\left(\mathrm{Vt}_{\mathrm{j}}\right)=-9,759636427966+2,076507841267 \cdot \operatorname{Ln}\left(\mathrm{D}_{(0,3 \mathrm{~m})}+0,900209471681 \cdot \operatorname{Ln}\left(\mathrm{Ht}_{\mathrm{j}}\right)\right.$ & 20.19 & 0.9933 \\
\hline 2 & $\operatorname{Ln}\left(\mathrm{Vt}_{\mathrm{j}}\right)=-9,909647463676+1,021064647448 \cdot \operatorname{Ln}\left(\mathrm{D}_{(0,3 \mathrm{~m})}{ }^{2} \cdot \mathrm{Ht}_{\mathrm{j}}\right)$ & 22.00 & 0.9932 \\
\hline 3 & $\operatorname{Ln}\left(\mathrm{Vt}_{\mathrm{j}}\right)=-9,593402231938+2,044167991731 \cdot \operatorname{Ln}\left(\mathrm{DEq}_{\mathrm{j}}\right)+0,945311495913 \cdot \operatorname{Ln}\left(\mathrm{Ht}_{\mathrm{j}}\right)$ & 22.02 & 0.9931 \\
\hline 4 & $\operatorname{Ln}\left(V_{j}\right)=-9,679267072736+1,012646004747 . \operatorname{Ln}\left(\mathrm{DEq}_{\mathrm{j}}^{2} . \mathrm{Ht}_{\mathrm{j}}\right)$ & 21.01 & 0.9931 \\
\hline 5 & $\mathrm{Vt}_{\mathrm{j}}=0,0000445895955929 .\left(\mathrm{DEq}_{\mathrm{j}}{ }^{1,852756766877}\right) \cdot\left(\mathrm{Ht}_{\mathrm{j}}^{1,409739765278}\right)$ & 18.46 & 0.9886 \\
\hline & $\mathrm{Vt}_{\mathrm{i}}=0,0000886865156307 \cdot\left(\mathrm{D}_{0,3 \mathrm{~m}}, 957072874247\right) \cdot\left(\mathrm{Ht}_{\mathrm{i}}^{0,870555587121}\right)$ & 17.66 & 0.9896 \\
\hline
\end{tabular}

Em que: Syx\% - erro padrão da estimativa em porcentagem, $R^{2} \mathrm{Aj}$. - $\mathrm{R}^{2}$ Ajustado.

variável independente diâmetro na base a $(0,3 \mathrm{~m})$ de altura (Figuras a e b), apresentaram os melhores ajustes. Para o modelo I, resíduos positivos iguais ou inferiores a $32,1 \%$ e resíduos negativos iguais ou superiores a $23,56 \%$ e, para modelo II, resíduos positivos iguais ou inferiores a $29,93 \%$ e resíduos negativos iguais ou superiores a $23,78 \%$. Por outro lado, $86,22 \%$ de fustes da equação resultante do Modelo I (Figura a) e $84 \%$ de fustes da equação resultante do Modelo II (Figura b) apresentaram estimativas de distribuição gráfica dos resíduos percentuais inferiores a $20 \%$.

Para a distribuição dos resíduos percentuais, quando das estimativas de volume total, as equações resultantes dos modelos de Schumacher e Hall, Modelo III e Spurr, Modelo IV, na forma logaritmizada, com a opção para a variável independente diâmetro equivalente $\mathrm{DEq}$, (Figuras c e d), apresentaram, para o modelo III, resíduos positivos iguais ou inferiores a $37,72 \% \mathrm{e}$ resíduos negativos iguais ou superiores a $36,36 \%$. Para o modelo IV, apresentaram resíduos positivos iguais ou inferiores a $36,12 \%$ e resíduos negativos iguais ou superiores a $35,58 \%$. Cerca de $88,88 \%$ de fustes do Modelo III (Figura c) e 89,23\% de fustes do Modelo IV (Figura d) apresentaram estimativas de distribuição gráfica dos resíduos percentuais inferiores a $20 \%$.

As equações resultantes para os modelos não lineares de Schumacher e Hall, com a opção para a variável independente diâmetro equivalente $\mathrm{DEq}$, modelo $\mathrm{V}$ e diâmetro na base a $(0,3 \mathrm{~m})$ de altura (Figuras e e f), apresentaram resíduos positivos iguais ou inferiores a $68,85 \%$ e resíduos negativos iguais ou superiores a $34,07 \%$. Para a equação VI, apresentaram resíduos positivos iguais ou inferiores a $62,24 \%$ e resíduos negativos iguais ou superiores a $21,68 \%$.

Em resumo, as equações resultantes dos modelos I, II, III e IV apresentaram resíduos uniformemente distribuídos sem nenhuma tendência clara de sub ou superestimação. As equações não lineares V e VI, (Figuras e e f), apresentaram as piores distribuições de resíduos, nos seus respectivos gráficos com tendência de superestimação dos volumes, notadamente nas primeiras classes diamétricas.

Araújo et al. (2004), trabalhando com a Mimosa tenuiflora e variáveis DAP $1,3 \mathrm{~m}$ e altura total, encontraram melhor ajuste para o modelo de Spurr. No presente trabalho, o uso das variáveis DEq 1,3 m e altura total proporcionaram resultados semelhantes, ainda que o ajuste tenha sido feito para dez espécies florestais existentes no Bioma Caatinga.

A utilização do diâmetro ao nível do solo DAS e DEq 1,30 m para o modelo de Schumacher e Hall modificado, permitiu estimar com precisão o volume total com casca dos fustes de espécies florestais presentes na caatinga (SILVA, 2005). Segundo este autor, o uso das equações estará na comodidade ou facilidade para aplicação em trabalhos de inventário florestal, medir o DAS ou DAB $0,3 \mathrm{~m}$ implica em se curvar até próximo ao nível do solo para coletar a informação, enquanto medir o DAP $1,3 \mathrm{~m}$ e posteriormente estimar o DEq 1,3 m o técnico trabalhará de pé, sem maiores esforços ergonômicos. 

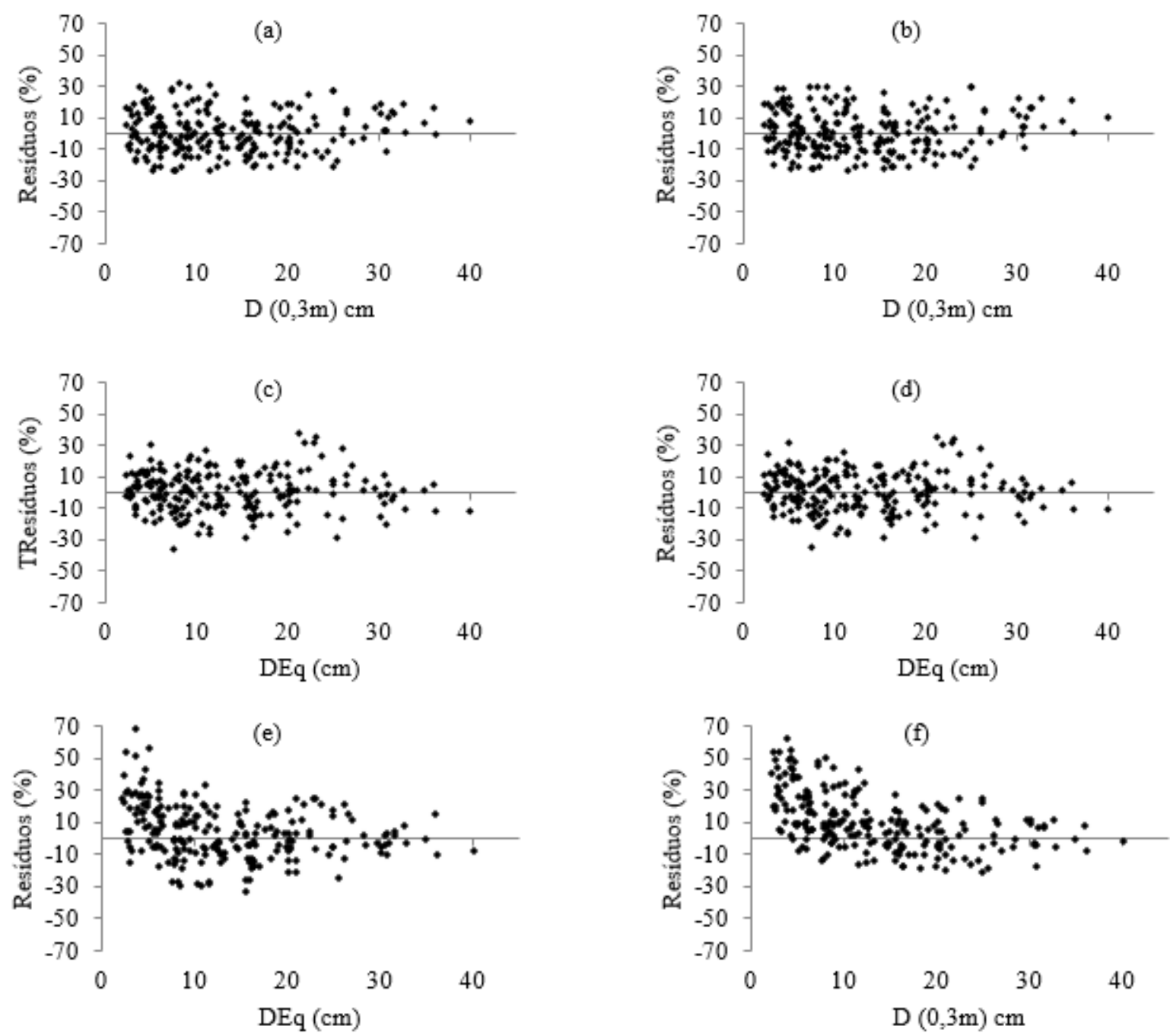

FIGURA 1: Distribuição dos resíduos percentuais para as estimativas de volume total, em ordem crescente de diâmetro (0,3 m), onde (a) com a equação I, (b) com a equação II, (c) com a equação III, (d) com a equação IV, (e) com a equação $\mathrm{V}$ e (f) com a equação VI.

FIGURE 1: Residual percentage distribution to estimate total volume, in order of growing diameter $(0,3 \mathrm{~m})$, where (a) with the equation I, (b) with the equation II, (c) with the equation III, (d) with the equation IV, (e) with the equation V and (f) with the equation VI.

\section{Fator de Forma}

O fator de forma com casca obtido com os dados da cubagem de 225 fustes abatidos para 10 espécies da caatinga (Anadenanthera colubrina, Aspidosperma pyrifolium, Bauhinia cheilantha, Combretum sp., Croton blanchetianus, Erythroxylum pungens, Handroanthus impetiginosus, Mimosa ophthalmocentra, Mimosa tenuiflora e Poincianella pyramidalis), produziu um fator médio igual a 0,871 , valor similar aos encontrados em outros estudos para a vegetação de caatinga por Silva (2005), Zakia et al. (1992) e Ferraz (2011) com 0,82, 0,9 e 1,06, respectivamente.

As espécies com maior número de fustes e que apresentaram elevados valores de fator de forma foram: Anadenanthera colubrina $(0,93)$, Poincianella pyramidalis (0,873), Mimosa tenuiflora $(0,867)$, Croton blanchetianus $(0,845) \mathrm{e}$ Aspidosperma pyrifolium $(0,893)$.

$\mathrm{O}$ fator de forma distribuído por classe diamétrica obteve as seguintes estimativas: classe I $(0,80)$, classe II $(0,869)$, classe III $(0,89)$, classe IV $(0,91)$, classe V $(0,86)$, classe VI $(0,86)$, classe VII $(0,938)$ e classe VIII $(0,857)$. As classes diamétricas III e IV apresentaram valores de fator de forma 
mais próximos do valor utilizado em vegetação de caatinga, que é de 0,9 (ZAKIA et al., 1992).

Silva (2005) propôs, para a sua área de estudo, o uso de dois valores de fator de forma, o primeiro para fustes com diâmetro menor que 20 $\mathrm{cm}$ ao nível do solo (classes 1 a 4), e o segundo fator de forma para fustes com diâmetro maior ou igual a $20 \mathrm{~cm}$ ao nível do solo (classes 5 a 7). Neste estudo foram encontradas duas estimativas de fator de forma para o cálculo de volume individual de fuste: diâmetro $<5 \mathrm{~cm}$ a $0,3 \mathrm{~m}$ de altura (classe 1), o fator de forma médio igual 0,80 e o diâmetro $\geq 5$ $\mathrm{cm}$ a $0,3 \mathrm{~m}$ de altura (classes 2 a 8 ), o fator de forma médio igual a 0,885 . Estes valores encontrados são semelhantes aos encontrados em estudos similares na vegetação da caatinga (ZAKIA et al., 1992; SILVA, 2005; FERRAZ, 2011).

O fator de forma torna-se uma técnica de grande importância para a obtenção de estimativas rápidas da variável dependente volume, estes autores ainda ressaltam que a sua precisão é inferior às equações de volume, sendo assim, deve-se ter ponderações e cuidados quanto a sua utilização (MIGUEL et al., 2010).

Os resultados obtidos no presente estudo permitiram gerar novos conhecimentos sobre vegetação de caatinga, dada a importância dos mesmos para a utilização de recursos florestais, sugere-se que estudos similares possam ser desenvolvidos em diferentes fisionomias deste bioma.

\section{CONCLUSÕES}

A área estudada possui elevada densidade e baixa riqueza de espécies. Além da maioria das espécies serem típicas do bioma a espécie Luetzelburgia bahiensis teve sua primeira ocorrência registrada para o estado da Paraíba.

Os fustes apresentaram-se em sua maior parte como saudáveis, mas com grande porcentagem de bifurcação, trifurcação e demais emissões e tortuosidade abaixo da altura comercial, características estas que indicam os usos mais diretos na forma de lenha, estacas e mourões.

Os modelos utilizados de Schumacher e Hall e Spurr, na forma logaritmizada, mostraram boas estimativas de volume de fustes da caatinga, apresentando-se como recomendáveis para vegetações que apresentem características semelhantes à vegetação em estudo.

Sugere-se o uso de dois fatores de forma para o cálculo de volume individual de fustes.

\section{AGRADECIMENTOS}

A CAPES, pela concessão da bolsa de estudos em nível de mestrado ao primeiro autor e ao INCRA e SUDEMA do estado da Paraíba pela permissão em desenvolver esta pesquisa na área de estudo.

\section{REFERÊNCIAS BIBLIOGRÁFICAS}

ALMEIDA NETO, J. X. et al. Composição Florística, Estrutura e Análise Populacional do Feijão-Bravo (Capparis flexuosa L.) no Semiárido Paraibano, Brasil. Revista Caatinga, Mossoró, v. 22, n. 4, p. 187-194, 2009.

ALVES JUNIOR, F. T. Estrutura, biomassa e volumetria de uma área de caatinga, florestaPE. 2010. 151 f. Tese (Doutorado) - Universidade Federal Rural de Pernambuco, Recife, 2010.

ALVES JUNIOR, F. T. et al. Estrutura diamétrica e hipsométrica do componente arbóreo de um fragmento de mata atlântica, Recife-PE. Cerne, Lavras, v. 13, n. 1, p. 83-95, 2007.

ANDRADE, M. V. M. et al. Levantamento Florístico e Estrutura Fitossociológica do Estrato Herbáceo e Subarbustivo em Áreas de Caatinga no Cariri Paraibano. Revista Caatinga, Mossoró, v. 22, n. 1, p. 229-237, 2009.

ARAÚJO, L. V. C. Composição florística, fitossociologia e influência dos solos na estrutura da vegetação em uma área de caatinga no semiárido paraibano. 2007.121 f. Tese(Doutorado) - Universidade Federal da Paraíba, Areia, 2007.

ARAÚJO, L. V. C. de et al. Estimativa da produção de biomassa de um povoamento de jurema-preta (Mimosa tenuiflora (Willd.) Poiret. com cinco anos de idade. Biomassa \& Energia, Viçosa, v. 1, n. 4, p. 347-352, 2004.

BELTRÃO, B. A. et al. Diagnóstico do município de São José de Espinharas, estado da Paraíba. 2005. Disponível em: <http://www.cprm.gov.br/ rehi/atlas/paraiba/relatorios/SAOJ182.pdf $>$. Acesso em: 21 set. 2014.

BFG. Growing knowledge: an overview of Seed Plant diversity in Brazil. Rodriguésia, Rio de Janeiro, v. 66, n. 4, p. 1085-1113. 2015.

CAMPOS, J. C. C.; LEITE, H. G. Mensuração florestal: perguntas e respostas. 3. ed. Viçosa: UFV, 2009. $548 \mathrm{p}$.

CHICHORRO, J. F. et al. Equações de volume e 
de taper para quantificar multiprodutos da madeira em floresta atlântica. Revista Árvore, Viçosa, v. 27, n. 6, p. 799-809, 2003.

EMBRAPA SOLOS. Levantamento Exploratório - Reconhecimento de solos do Estado da Paraíba. UEP Recife, 2006. Disponível em: <http://www. uep.cnps.embrapa.br/solos/index.php?link=index $>$. Acesso em: 20 dez. 2014.

FERRAZ, J. S. F. Análise da Vegetação de Caatinga Arbustivo-Arbórea em Floresta, PE, como Subsídio ao Manejo Florestal. 2011. $131 \mathrm{f}$. Tese (Doutorado) - Universidade Federal Rural de Pernambuco, Recife, 2011.

FUNDAÇÃO DE CIÊNCIA E TECNOLOGIA (RS). Software Mata Nativa 2: manual do usuário. Viçosa: Cientec, 2006. 295 p.

GUEDES, R. S. et al. Caracterização florísticofitossociológico do componente lenhoso de um trecho de caatinga no semiárido paraibano. Revista Caatinga, Mossoró, v. 25, n. 2, p. 99-108, 2012.

IBAMA. Monitoramento do Desmatamento nos Biomas Brasileiros por Satélite. Acordo de Cooperação Técnica MMA/IBAMA. Monitoramento do Bioma Caatinga 2002 a 2008. CSR/IBAMA, 2010. Disponível em: <http://www. mma.gov.br/estruturas/sbf chm rbbio/ arquivos/ relatrio_tcnico_caatinga_72.pdf $>$. Acesso em: 20 $\operatorname{dez} 2014$.

LEITE, J. A. N. Análise quali-quantitativa da vegetação arbustivo-arbórea da caatinga, em Teixeira-PB. 2010. 56 f. Dissertação (Mestrado) Universidade Federal de Campina Grande, Patos, 2010.

MARANGON, L. C. et al. Estrutura fitossociológica e classificação sucessional do componente arbóreo de um fragmento de floresta estacional semidecidual, no município de Viçosa, Minas Gerais. Cerne, Lavras, v. 13, n. 2, p. 208-221, 2007.

MIGUEL, E. P. et al. Ajuste de modelo volumétrico e desenvolvimento de fator de forma para plantios de Eucalyptus grandis localizados no município de Rio Verde-GO. Enciclopédia Biosfera, Goiânia, v. 6, n. 11, p. 1-13, 2010.

OLIVEIRA, P. T. B. de. et al. Florística e Fitossociologia de Quatro Remanescentes Vegetacionais em Áreas de Serra no Cariri Paraibano. Revista Caatinga, Mossoró, v. 22, n. 4, p. $169-178,2009$.
REDE DE MANEJO FLORESTAL DA CAATINGA. Protocolo de Medições de Parcelas Permanentes. Recife: MMA; PNF; APNE, 2005. $28 \mathrm{p}$.

REVEAL, J. L.; CHASE, M. W. APG III: Bibliographical information and Synonymy of Magnoliidae. Phytotaxa, Nova Zelândia, v. 19, p. 71-134. 2011.

RODAL, M. J. N. et al. Estrutura da vegetação caducifólia espinhosa (Caatinga) de uma área do sertão central de Pernambuco. Hoehnea, Água Funda, v. 35, n. 2, p. 209-217, 2008.

SAMPAIO, E. V. S. B. et al. Tree biomass estimation in regenerating areas of tropical dry vegetation in northeast Brazil. Forest Ecology and Management, Amsterdam, v. 259, p. 1135-1140, 2010.

SANTANA, J. A. S.; SOUTO, J. S. Diversidade e estrutura fitossociológica da caatinga na Estação Ecológica do Seridó-RN. Revista de Biologia e Ciências da Terra, Campina Grande, v. 6, n. 2, p. 232-242, 2006.

SCOLFORO, J. R. et al. A. Obtenção de relações quantitativas para estimativa de volume do fuste em floresta estacional semidecídua Montana. Cerne, Lavras, v. 1, n. 1, p. 123-134, 1994.

SILVA, J. A. A. et al. Modelagem volumétrica de Pinus elliottii Engelm., no Planalto do estado de Santa Catarina. Revista Árvore, Viçosa, v. 24, n. 1, p. 91-96, 2000.

SILVA, J. A. Fitossociologia e relações alométricas em caatinga nos estados da Paraíba e Rio Grande do Norte. 2005. 93 f. Tese (Doutorado) - Universidade Federal de Viçosa, Viçosa, 2005.

SOARES, C. P. B. et al. Dendrometria e inventário florestal. Viçosa: UFV, 2006. 276 p.

THAINES, F. et al. Equações para estimativa de volume de madeira para a região da bacia do Rio Ituxi, Lábrea, AM. Boletim de Pesquisa Florestal, Colombo, v. 30, n. 64, p. 283-289, 2010.

UNIVERSIDADE FEDERAL DE CAMPINA GRANDE. Departamento de Ciências Atmosféricas. Dados Climatológicos do Estado da Paraíba. 2014. Disponível em: <http:// www.dca.ufcg.edu.br/clima/dadospb.htm>. Acesso em: 24 set. 2014.

VELOSO, H. P. et al. Classificação da vegetação brasileira, adaptada a um sistema universal. Rio 
de Janeiro: IBGE, 1991. 124 p.

ZAKIA, M. J. B. et al. Equações de Peso e volume para oito espécies lenhosas nativas do SeridóRN. Natal: PNUD/FAO/IBAMA, 1992. v. 1. 